\title{
Palliative Care Initiation in Chronic Obstructive Pulmonary Disease: Prognosis-Based, Symptoms-Based or Needs-Based?
}

This article was published in the following Dove Press journal: International Journal of Chronic Obstructive Pulmonary Disease

\author{
Ruxandra-Mioara \\ Rajnoveanu' \\ Armand-Gabriel \\ Rajnoveanu ${ }^{2}$ \\ Ariadna Petronela Fildan ${ }^{3}$ \\ Doina Adina Todea $\mathbb{D}^{\prime}$ \\ Milena Adina Man' \\ Nicoleta Stefania Motoc (D) \\ Daniela Mosoiu ${ }^{4}$ \\ 'Pneumology Department, luliu \\ Hatieganu University of Medicine and \\ Pharmacy, Cluj-Napoca, Romania; \\ ${ }^{2}$ Occupational Medicine Department, \\ Iuliu Hatieganu University of Medicine \\ and Pharmacy, Cluj-Napoca, Romania; \\ ${ }^{3}$ Pneumology Department, Faculty of \\ Medicine, Ovidius University, Constanta, \\ Romania; ${ }^{4}$ Hospice Casa Sperantei \\ Brasov, Transilvania University, Brasov, \\ Romania
}

Correspondence: Armand-Gabriel Rajnoveanu

Occupational Medicine Department, luliu Hatieganu University of Medicine and Pharmacy, Cluj-Napoca 400365, Romania

Tel +40740770505

$\mathrm{Fax}+40264597453$

Email armand.rajnoveanu@umfcluj.ro
Abstract: The absence or late initiation of palliative care (PC) in chronic obstructive pulmonary disease (COPD) is multidimensional. To provide palliative care from the moment of COPD diagnosis remains utopic. Even the advanced forms or the end-oflife stages benefit late or never from these services. In this context, the research questions for the present systematic review were focused on the prognosis variables or multicomponent indices in COPD patients alongside the symptoms and unmet needs, which may be useful for the palliative care initiation. The aim was to help clinicians to identify not only the tools reliable to predict poor survival in COPD patients but also to identify the criteria for appropriateness for early palliative care onset. The search included systematic reviews and reviews published in English in the PUBMED database from Jan 1, 2015 to Jan 6, 2020. From a total of 202 findings, after applying filters, using additional sources, and eliminating duplicates, the search strategy screened 16 articles, out of which 10 were selected and included. A Preferred Reporting Items for Systematic Review and Meta-analysis Protocols (PRISMA-P) flow diagram was constructed. The main domains identified as barriers in providing palliative care in COPD patients were complex: from the prognosis difficulties to the prognostic variables and scores proposed for initiating PC; from the troublesome symptoms or the unidimensional symptom tools to the unmet needs of COPD patients. The review concluded that none of the existing prognostic variables and multicomponent indices are reliable enough to exclusively predict poor survival in COPD patients and the decision to initiate PC should be rather based on the presence of refractory symptoms and patients' unmet needs and preferences. Despite the current advances, the ideal model to initiate palliative care from the moment COPD is diagnosed is a goal for clinicians trained in, and capable of providing palliative care in any COPD patient.

Keywords: COPD, palliative, care, prognosis, symptom, needs, unmet

\section{Introduction}

Chronic respiratory diseases (CRDs) are a group of chronic diseases that affect the airways and other structures of the lung, according to the definition of the World Health Organization (WHO). ${ }^{1}$ Both the prevalence and the global burden of CRDs are increasing worldwide, with an important impact on the quality of life. ${ }^{2}$ Even though CRDs are not curable, the WHO Global Alliance against CRDs (GARD) focuses on the prevention of these diseases and care of these patients worldwide, in order to globally reduce morbidity, disability, and premature mortality related to the diseases. 
Under the umbrella term of CRD, one of the most common diseases included is represented by chronic obstructive pulmonary disease (COPD). ${ }^{1}$ It is considered a public health challenge as a major contributor to global morbidity and mortality. ${ }^{3}$ Currently, COPD is the fourthleading global cause of death, and in 2030 it is expected to become the third cause of mortality ${ }^{4}$ and the seventh leading cause of disability-adjusted life years (DALYs) lost worldwide. ${ }^{5}$

Alongside interstitial lung disease (ILD) and bronchiectasis, COPD is a Non-Malignant Respiratory Disease (NMRD), another umbrella term proposed by the National End of Life Care Intelligence Network in 2011. ${ }^{6}$ Even though, worldwide, over 210 million people have a diagnosis of COPD, and millions of others have another form of NMRD, ${ }^{7}$ a holistic approach to the diseases is still an idealistic concept globally. Palliative care is a patientcentered holistic approach, focused on the management of symptoms, good communication between patients and health-care providers, and maintaining quality of life ${ }^{8}$ of patients and their family, through the prevention and relief of suffering. According to the World Health Organization (WHO), PC improves the quality of life by early identification and impeccable assessment and treatment not only of pain and other overwhelming symptoms but also addressed to the psychological, social, and spiritual dimensions. ${ }^{9,10}$

From this point of view, the role of PC for patients with NMRD was emphasized by the key international respiratory guidelines. ${ }^{11}$ The American Thoracic Society (ATS) highlighted the importance of integrating palliative care from the moment of diagnosis. ${ }^{12}$ Regardless of the unpredictable course of the disease, PC should be encouraged to occur early in the disease trajectory. ${ }^{11}$ Still, the estimated need for palliative care in patients with chronic respiratory diseases is high $(10.3 \%)$, coming second to that in cardiovascular diseases $(38.5 \%)$ and cancer $(34 \%){ }^{13}$ Unfortunately, palliative care is not available worldwide for these patients, independently of what settings are considered. The possible explanations and barriers for this fact are complex and found even in countries where the role and development of palliative care services are worked out for many years. The aim of the current review was to offer an overview of the latest data about the initiation of palliative care in patients living with COPD, from the existing prognosis and multivariable scores to the burden of refractory symptoms and the unmet needs of patients and those close to them. The purpose was to help clinicians to identify not only the tools reliable to predict poor survival in COPD patients but also to identify the criteria for appropriateness for early palliative care onset.

\section{Research Questions}

How should palliative care be initiated in COPD patients: prognostic-based, symptoms-based, and/or needs-based?

\section{Materials and Methods}

\section{Inclusion/Exclusion Criteria of the Studies}

The inclusion criteria were articles related to palliative care and the prognosis in COPD. Other inclusion criteria were: studies focused on the troublesome symptoms of COPD patients, studies about the unmet needs of patients living with COPD and palliative care. The main included article types were reviews and systematic reviews.

The exclusion criteria were: studies not related to the topic/methodology; articles related to other diseases not to COPD; articles addressed only to lung cancer patients; articles not related at all to the palliative care perspective; studies with only abstracts available; case-studies; randomized control studies; duplicates.

\section{Literature Search}

This systematic review was based on the search from electronic data sources using the PubMed (MEDLINE) database. There was no need for Ethics Committee approval since this study was a synthesis of published studies. The initial search strategy on the keywords Palliative Care and Prognosis in COPD identified 202 results. After applying the filters: Systematic Reviews, Review, Full text, published in the last 5 years, Humans, English, 16 records were identified. The publication date of the search was between Jan 1, 2015 and Jan 6, 2020. The search included full-text systematic reviews and reviews published in the English language. We supplemented our electronic database search from other sources, including reference lists of included studies, index-related articles on PubMed, and existing relevant reviews. Four additional records were identified from other sources. From the 20 studies identified, after excluding the 4 duplicates, 16 records were screened. After further evaluations of the title and abstracts, 2 studies were excluded. From the 14 full-text articles assessed for eligibility, 4 articles were excluded because they were not related to the topic. Finally, 10 articles were included in our review: 4 systematic reviews ( 1 with validation cohort), 5 reviews, and 
1 cohort study. A manual search was performed afterward. We were initially focused on the studies involving palliative care onset in COPD mainly from the prognostic perspective, but we further extended our analysis on the importance of refractory symptoms and the unmet needs of COPD patients. A Preferred Reporting Items for Systematic Review and Meta-analysis (PRISMA) ${ }^{14}$ flow diagram was constructed, as shown in Figure 1. The screening of the English titles and abstracts of the articles with potential eligibility was also simultaneously conducted by one independent researcher (AGR). The two reviewers read and analyzed all full-text articles, considering the inclusion and exclusion criteria. Any differences in opinion were discussed and resolved by consensus. A narrative synthesis of the identified evidence was performed.
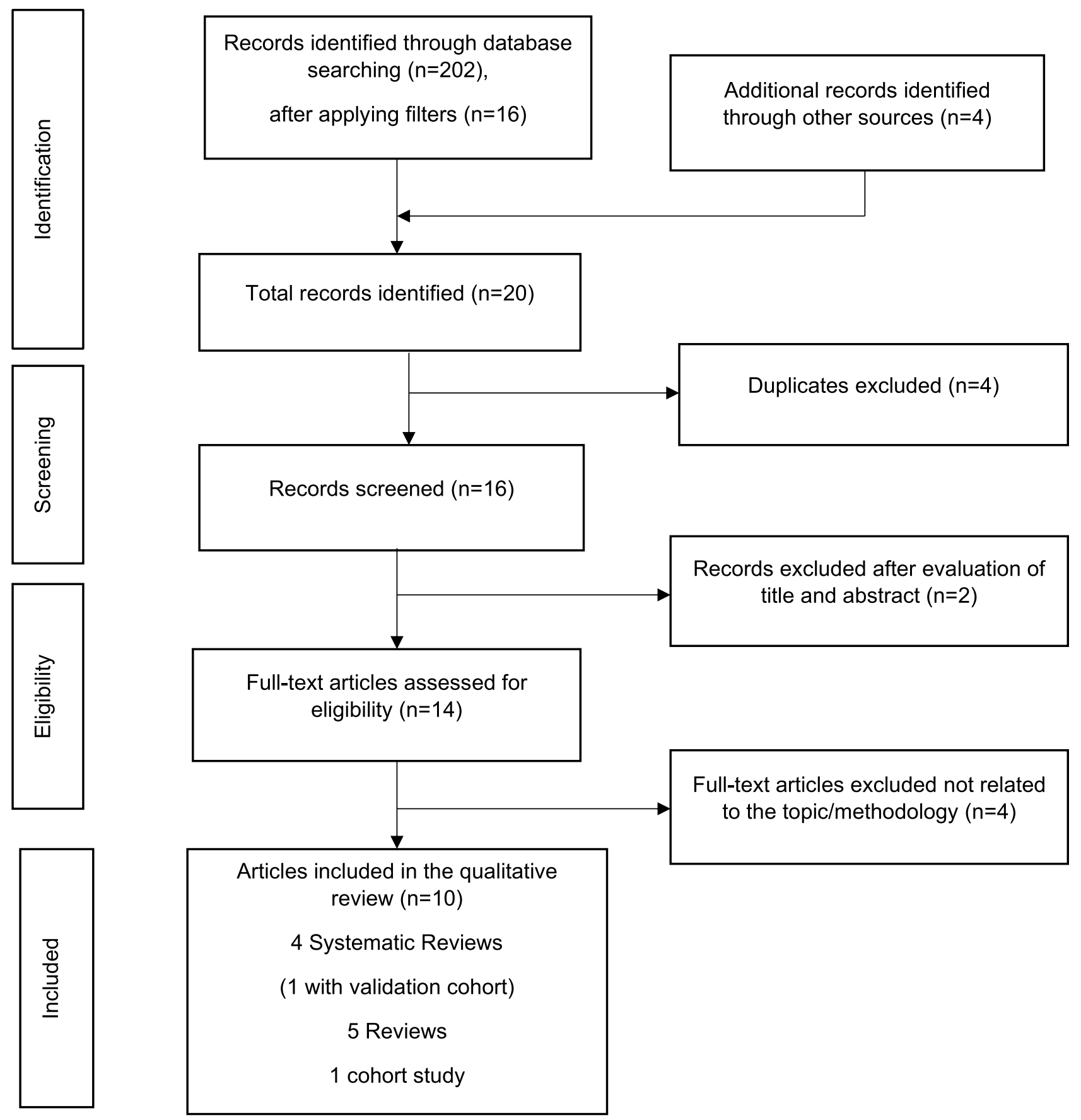

Figure I Literature identification process (PRISMA flowchart). 


\section{Results}

After reading and analyzing the included studies, some issues emerged. A brief synthesis of the reviewed articles with their main findings, identified gaps and limitations are summarized in the Supplementary materials Table S1.

\section{Difficulties of Prognosis - an Important}

\section{Barrier to Provide PC}

The unpredictable and prolonged trajectory of the disease makes prognostication difficult for physicians, patients, and their families. ${ }^{15}$ The inexact prognosis of this chronic life-limiting illness, with a progressive decline punctuated with acute, potentially life-threatening exacerbations, is not only a clinical challenge but also a barrier to timely referral and receipt of palliative care. ${ }^{3,16}$ The questions about the moment to start PC and which COPD patients should receive it remain controversial. ${ }^{17}$ In their systematic literature review of 17 European guidelines and 2 pathways, Siouta et al offered an overview of the level of integration of PC in COPD. ${ }^{18}$ The determination of the referral criteria to start $\mathrm{PC}$, the moment to initiate $\mathrm{PC}$, and the modest predictive value of different prognostic tools were debatable topics analyzed in the systematic review. Though 12 out of the 19 guidelines/pathways explicitly discussed the point of which PC should be initiated, large variations of the exact timing to start PC were noted: from the moment of COPD diagnosis or only for the terminally ill stages or depending on the frequency of exacerbations or hospitalizations, to the last 12 months of life or to the last 6 months of life (3 guidelines from the Netherlands, but none of them invoked the surprise question). ${ }^{18}$ Besides the above-mentioned aspects, the study highlighted the overall growing awareness for the importance of integrated PC in advanced forms of COPD patients, although a standardized and universally accepted definition of integrated $\mathrm{PC}$ is missing. ${ }^{18}$ A possible solution to overcome these barriers is to include individualized palliative interventions in the management of COPD patients from the moment of diagnosis. Still, the ideal model proposed for early integrated PC in daily practice needs further validation, as Harrington et al outlined. ${ }^{16}$

\section{Prognostic Variables and Scores Proposed for Initiating PC}

Significant factors in prognosticating are available. ${ }^{19}$ there is a lack of a "gold standard" method to predict prognosis ${ }^{5}$ and consequently to integrate palliative care as early as possible. Starting from the observation that current recommendations to consider initiation of PC in COPD are often based on an expected poor prognosis ( $\leq 1$ year survival) ${ }^{17}$ in their systematic review Almagro et al analyzed the reliability of 17 prognostic variables and 3 multicomponent indices recommended for start PC based on an expected 1-year mortality. The utility of the proposed variables was further validated in a cohort study of 697 patients hospitalized for COPD exacerbation. Ten predictive mortality variables with statistical significance ( $p$-value $\leq 0.05$ ) were identified. Among them, only 4 had a concomitant sensibility and specificity $\geq 50 \%$ : age $\geq 70$ years, severe dyspnea (3-4 in the modified dyspnea Medical Research Council (mMRC)), depression (Yesavage scale $\geq 5$ points), and poor health-related quality of life (Saint George's Respiratory Questionnaire $\geq 60$ points). The other predictive mortality variables that reached statistical significance were: severe physical dependence with loss $\geq 3$ activities of daily living (Katz index), low body mass index (BMI $\leq 21 \mathrm{~kg} / \mathrm{m}^{2}$ ), the presence of comorbidities (Charlson index $\geq 3$ ) and 3 multicomponent indices $\geq 7$, like the BODE index (Body mass index, Obstruction, Dyspnea and 6 min walking test), the BODEx index (Body mass index, airflow Obstruction, Dyspnea, severe exacerbations) and the CODEX index (Comorbidity, Obstruction, Dyspnea, severe exacerbations). Almagro also tested in the cohort study an adaptation of the Curtis criteria, ${ }^{20}$ which represent another combination of variables proposed as a main criterion to start PC based on a poor prognosis (forced expiratory volume in $1 \mathrm{~s}$ (FEV1) $<30 \%$ predicted; oxygen dependence; $\geq 1$ hospital admissions in the previous year for an acute exacerbation of COPD; left heart failure or other comorbidities; weight loss or cachexia; decreased functional status; increasing dependence on others; age $>70$ years). The only statistically significant cut-off for this criterion was $\geq 3$ (61\% patients), ${ }^{17}$ not $\geq 2$ as recommended by Curtis. $^{20}$

Summarizing the results, the identified variables and multicomponent indices may be considered useful predictors of survival, but they should not be used as exclusive criteria for initiating PC, because none of them is sufficiently reliable. The timing to start PC in COPD patients should be based on the presence of symptoms refractory to conventional treatment, together with patients' preferences. ${ }^{17}$ Prognostic variables play rather a secondary role. Smith et $\mathrm{al}^{5}$ highlighted the same 
conclusion in their systematic review. They did not find a single variable or a multivariate score that can be recommended for use in practice to predict mortality at or less than 1 year. Among the 6 multivariable and the 3 comorbidity indices analyzed, only one score was derived identified - the B-AE-D score: ${ }^{21}$

$\mathrm{B}$ - body mass index [BMI]

$\mathrm{AE}$ - severe acute exacerbation of COPD [AECOPD] frequency

D - modified Medical Research Council [mMRC] dyspnea severity.

The results completed the findings of the Almagro study, ${ }^{17}$ because they were obtained from stable COPD patients (not hospitalized). ${ }^{5}$ Smith et al also noted, but did not include in their review, four tools for the identification of COPD patients in the last year of life: the Gold Standards Framework Prognostic Indicator Guidance (GSF-PIG), the RADboud Indicators of Palliative Care Needs (RAD-PAC), the Supportive and Palliative Care Indicators Tool (SPICT), and the Necesidades Paliativas (NECPAL) program. The mentioned tools were developed using expert knowledge, based on the existing studies, combined with the clinical experience. However, the major limitation of these tools is that they were not tested for accuracy and no evidence of reliable prognostication prediction or about other outcomes is available. They also may not be compared to other multivariable tools derived using statistical methods. Those were the main motifs for not being included in their review. ${ }^{5}$ Recently, in 2019, Bloom et al derived a prognostic risk model to predict mortality at 1 year based on 18 different variables - the BARC index, acronym derived from: ${ }^{22}$

B - body mass index and blood tests,

A - age,

$\mathrm{R}$ - respiratory variables (airflow obstruction, exacerbations, smoking),

$\mathrm{C}$ - comorbidities.

The Clinical Practice Research Datalink (CPRD) was used as a data source for the COPD populations. Though the model was aimed to increase predictive performance and to offer clinical and practical advantages, the usefulness in primary care or in hospitalized patients seems equivocal. The excessive number (18) of incorporated variables, the lack of specification for the cut-off points and scores of each variable limit the applicability for clinicians.

\section{Initiating Palliative Care in COPD \\ Patients - More Symptom-Based, Than Prognostic-Based}

People with advanced COPD experience distressing physical and psychological symptoms together with a lot of concerns, ${ }^{3}$ expressed more or less. The importance of troublesome symptoms like dyspnea, fatigue, cough, cachexia, depression, or anxiety, that can occur during the course of the disease, was emphasized by literature in recent years. ${ }^{3,15,23}$ Besides their high prevalence and prognostic implications, their impact on the quality of life is significantly experienced by COPD patients. ${ }^{17}$ Moreover, they also have an important psychosocial effect. For example, severe breathlessness causing functional disability may contribute to the patient's isolation and loneliness. ${ }^{3}$ Refractory symptoms that persist despite optimal management of the underlying diseases must be recognized by clinicians. The detailed medical history, together with comprehensive symptom assessment, including the evaluation of the level of dyspnea or other existing symptoms, their evolution during the course of the disease, and the diagnosis of their underlying factors, have to be carefully evaluated. It is not limited to the severity of symptoms but also includes the multifaceted effect of symptoms on patients and their caregivers' life. $^{3}$

The focus on the assessment and the management of patients' bothersome symptoms and concerns ${ }^{3}$ should be a priority in clinical practice in order to establish the need for initiating PC according to the patient's own goals. In this context, integrated palliative care should be considered alongside the usual therapy, the non-pharmacological and the psychological measures, ${ }^{17}$ in order to reduce suffering, ${ }^{18}$ by improving the symptoms and the quality of life, ${ }^{3,15}$ to reduce the health-care utilization and to increase the patient and caregiver satisfaction. ${ }^{3}$

Refractory breathlessness related to deconditioning, increased depression and anxiety, and impaired quality of life is also associated with a higher risk of hospitalization and premature mortality. ${ }^{23}$ In order to better assess the dimensions of breathlessness in different settings of COPD population, uni- and multidimensional tools were developed and validated. Ekstrom et al reviewed recent advances in the outcome measurement of refractory breathlessness in COPD patients. ${ }^{23}$ Two validated unidimensional tools were outlined. The Exacerbations of Chronic Pulmonary Disease Tool (EXACT) Respiratory Symptoms (RS) (E-RS) ${ }^{24}$ can be used to 
measure the intensity of daily respiratory symptoms (breathlessness, cough, sputum, chest symptoms) as a summary score in daily life in patients with stable COPD. The other unidimensional tool that is reliable and responsive measures dyspnea during daily activities in COPD outpatients; this is the weekly mean SOBDA score (Shortness of Breath with Daily Activities). ${ }^{19,25}$ As the impact of breathlessness is complex, the use of multidimensional tools such as the Dyspnea- $12^{26}$ score or the Multidimensional Dyspnea Profile, ${ }^{27}$ aimed to measure the intensity, the unpleasantness, the quality, and the patients' emotional response $^{23}$ to it, can be a useful aid for the clinicians that treat these patients. As Maddocks et $\mathrm{al}^{3}$ highlighted in their review, most pharmacological approaches for breathlessness do not cover all the complex aspects related to this symptom. Though there is a limited evidence base, the use of pharmacological treatments together with non-pharmacological therapies, like pulmonary rehabilitation, component interventions (breathing techniques, hand-held fan), cognitive behavioral therapy, or multi-professional integrated breathlessness services, showed benefits in patients experiencing dyspnea. ${ }^{3}$ If breathlessness is refractory, unremitting despite the optimal management, other drugs can be used. In these patients, a low dose of oral or parenteral opioids could palliate breathlessness, although the evidence base is of low quality, with few small trials and participants. Potential related side-effects such as constipation, nausea, or vomiting should be constantly identified, managed, and explained, ${ }^{3}$ as they can be a reason for opioid's withdrawal and an additional source of concerns for patients and their families. In clinical practice, benzodiazepines may be considered as a second or third-line treatment for breathlessness which is not responding to opioids, though studies failed to support their use. Antidepressants may relieve dyspnea for two reasons: the implication of the serotonergic pathways and the fact that depression commonly occurs together with breathlessness. ${ }^{3}$ Though severe depressive symptoms or the clinical depression are present in almost $40 \%$ of COPD patients, ${ }^{19}$ they are difficult to diagnose in daily practice due to the symptoms overlapping between the two diseases. In this context, the use of the six-item Hamilton Depression Rating Subscale (HAM-D 6 ), ${ }^{19}$ a sensitive tool comparable to the original version, can be helpful for the screening of depression. This comorbidity (Yesavage scale $\geq 5$ points) was identified as a statistically significant predictive 1-year mortality variable in COPD patients ${ }^{17}$ with an important impairment on the quality of life. ${ }^{17,19}$ Complex interventions, including cognitive behavioral therapy, psychological and lifestyle interventions, multicomponent exercise training, and antidepressant drugs (selective serotonin-reuptake inhibitors best tolerated) have supporting therapeutic evidence. ${ }^{3}$ COPD patients may experience different pains, of several aetiologies, possibly caused by the systemic effects or therapies (corticosteroids use) of the disease or by the preexisting comorbidities. ${ }^{3}$ Non-opioids, opioids, or new analgesics are the pharmacological options that may be used inpain management. ${ }^{3}$ The assessment and treatment of the potentially adverse effects are mandatory. Daytime sleepiness and insomnia may be another bothersome symptom in the life of COPD patients. Good sleep hygiene, cognitive behavioral therapy, benzodiazepines, or non-invasive positive pressure ventilation for hypercapnic patients with stable disease may be used for the treatment, as Maddocks et al noted. ${ }^{3}$

\section{The Complex Unmet Needs of COPD Patients}

Due to the complexity of the disease and the multiple areas of needs, the preferences of COPD patients have to be actively assessed. The process should be ongoing, as the preferences of patients and their caregivers might change over time. ${ }^{3}$ In their systematic review of qualitative findings, Clari et al identified three main domains regarding the perception of the unmet needs:

1) unmet needs regarding information about the disease,

2) unmet physical, emotional, spiritual, financial, and social support needs, and

3) unmet care needs. ${ }^{4}$

Besides the sustained symptom burden, patients living with COPD often have a limited understanding of their disease. ${ }^{3}$ A reason for that may be the frequent communication barrier from both the patient and their doctor's perspective. ${ }^{15}$ The unmet needs around knowledge of COPD include: information regarding different potential risk factors such as cigarette smoking, the lack of understanding of the most-used terminology related to COPD, or the lack of data about the disease management, including self-management decisions and measures. ${ }^{4}$ Patients' concerns about not receiving information are also related to the prognosis and the progression of the disease, including the end-of-life stages. ${ }^{3,4}$ On the other hand, health-care professionals should take into account that not all patients 
and their caregivers are opened to end-of-life discussions; therefore, the assessment of individual communication preferences is extremely important. ${ }^{3}$

The troublesome symptoms of advanced COPD, like breathlessness, chest pain, fatigue, or the unwanted effects of treatments may compromise not only the physical needs, disrupting the daily living activities, but also the social needs of these patients. ${ }^{4}$ They may feel frustrated, anxious, depressed, angry, ${ }^{4}$ self-blaming, or even demeaned by the limitations of the disease. ${ }^{3}$ The fear and concerns about the future, the fear of dependence (on family, home oxygen), the feeling of being a burden for their families, and the reduced self-esteem, are other sources of their unmet emotional needs. ${ }^{3,4}$ It is important to identify and treat them, by offering psychological, dignity-conserving therapies and spiritual support. ${ }^{3}$ Due to the severe symptom burden, patients may become obliged to quit their jobs or pay for household help. ${ }^{3}$ Living with these constraints, together with the costs for home oxygen and medications, represent important unmet needs regarding financial support. ${ }^{3,4}$ Not only do COPD patients experience unmet needs, but also do their caregivers, family members, or those who are close to the patient. They may have their own needs and concerns. Therefore, to provide information about COPD and its management and to offer psychological care and support for the caregivers should not be neglected. ${ }^{3}$

People with COPD often experience difficulties in accessing health-care professionals and health-care services, including community services. ${ }^{4}$ The reasons are variable, from the scarce knowledge about the modalities and facilities to access the healthcare system in different situations, to the lack of communication between their family doctors and hospitals or the lack of dedicated services for transportation. ${ }^{4}$

Another important unmet need in the care of COPD patients is the late referral in providing integrated palliative care. There is an increasing awareness about the need to have a greater access to PC services. ${ }^{15,18}$ Still, patients living with advanced forms of COPD are quite unlikely to get engaged in discussions with their doctors about end-oflife issues and the opportunity to receive palliative care services, including advance care planning (ACP) discussions. ${ }^{16}$ Though studies on the effectiveness of $\mathrm{ACP}$ on subsequent care in COPD are limited, and future research is required, it showed improvement in the quality of communication. ${ }^{3}$ Despite the communication barriers between doctors and patients, highlighted by the most guidelines/pathways, the referral criteria and timings are not only widely different, but also equivocal because some of them were mainly based on prognostication. ${ }^{18}$ The wide variability and obstacles in accessing palliative care in different countries, including reimbursement issues, ${ }^{15}$ the insufficient time claimed by clinicians or their reduced communication skills, ${ }^{3}$ the focus especially on the terminal phases of the disease, ${ }^{15}$ the lack of PC trained health-care staff or doctors who are not prepared to provide trained palliative care services, are other potential motifs for the late initiation of PC. In order to overcome these various barriers, proactive palliative care approaches may be early incorporated in the management of COPD. ${ }^{15}$ The use of the "surprise question" can be a supplementary screening tool for the primary care physicians, to identify patients appropriate for proactive palliative care. ${ }^{15} \mathrm{PC}$ should not be limited to specialists in palliative care. Any physician involved in the care of patients living with COPD should be familiarized with PC. Primary care providers as well as pulmonologists should be trained and competent to identify the appropriateness of a patient who would benefit from proactive outpatient supportive care, as part of comprehensive COPD care. ${ }^{15}$ Regardless of the care models included in the proactive PC approaches, such as the proactive primary care approach proposed by Vermylen et $\mathrm{al}^{15}$ or the models of integrated PC outlined by Maddocks et al, all of them are focused more on the unmet needs of patients living with COPD, than on the prognosis. ${ }^{3,15}$ As potential models for integrative working with palliative care for COPD people, Maddocks et al proposed: refractory symptom triggered services (for example, for refractory breathlessness), short-term integrated PC (such as the Breathlessness Support Service), advanced COPD clinics, or Integrated Respiratory Care services (pulmonary rehabilitation, hospital at home). ${ }^{3}$

The involvement of multidisciplinary PC teams, including physicians, disease specialists, nutritionists, physiotherapists, nurses, psychologists, psychiatrists, occupational therapists, social workers, chaplains, etc., and personnel additionally trained in $\mathrm{PC},{ }^{3,15,18}$ was promoted in most of the guidelines/pathways, as noted by Siouta and colleagues. ${ }^{18}$ The global approach of the multidimensional unmet needs of COPD patients, with early, longitudinal palliative care provided by an interdisciplinary team, ${ }^{15}$ offers an improvement in the care of people living with COPD, including for those that do not correctly identify their needs. ${ }^{4}$ 


\section{Discussion}

The present review, like other reviews involving the topic of palliative care, ${ }^{18}$ included studies predominantly from the UK, probably because PC originated from the UK. ${ }^{28}$

Despite the small number of studies included in our search, some important issues emerged. The barriers to the initiation of palliative care in people living with COPD are multidimensional. The referral criteria and timing for $\mathrm{PC},{ }^{18}$ the appropriate identification of patients for referral to $\mathrm{PC}$, prognostic variables and scores, ${ }^{5,17}$ the settings, the staffing, and the models to provide $\mathrm{PC},{ }^{3,15}$ alongside the complex unmet needs of patients with $\mathrm{COPD},{ }^{3,4}$ are topics much debated in the literature, including in recent years. ${ }^{15,16,29}$

The unpredictability of the prognosis in an individual patient ${ }^{15}$ is one of the main obstacles claimed for timely initiation of PC. Given that prognostication is difficult and inexact, the determination of the referral criteria should be independent of it. ${ }^{18}$ Though there are more than 400 prognostic models, using a combination of different predictors for COPD related outcomes, a limited number were externally validated. ${ }^{30}$ On the other hand, to decide which prognostic model is better to choose in different settings, geographical area, and individuals with COPD, even if it was externally validated, may be a challenge for physicians in daily practice. ${ }^{30}$ Except for the updated 15-point ADO index ${ }^{31}$ and the model developed by Bertens et al, ${ }^{32}$ which were not explored in the studies included in our search, the other prognostic variables like ADO, B-AE-D, and the updated BODE were also analyzed in the systematic review of Bellou et al which showed a low risk of bias for these prognostic models. ${ }^{30}$ However, according to the data of Almagro ${ }^{17}$ and Smith $^{5}$ none of the prognostic variables and multicomponent indices that were identified offer sufficient reliability to predict 12 months of survival in COPD patients. ${ }^{5,17}$ A possible explanation is related to the fact that these prognostic factors are usually related to long-term survival; also, their applicability is hard to generalize in any COPD patient. ${ }^{17}$ The need to move from prognostic paralysis to active total care should be taken into consideration. ${ }^{33}$

The initiation of PC should not be based on life expectancy, ${ }^{17}$ but rather on refractory, uncontrolled symptoms like breathlessness, fatigue, cough, called sometimes the respiratory cluster, ${ }^{34}$ alongside the emotional, social, and spiritual needs and preferences of patients. ${ }^{3,4}$ The assessment of symptoms such as breathlessness is usually based on unidimensional validated tools or scales. ${ }^{23}$ Measurement focused only on intensity may overlook the multidimensional nature of symptoms and miss valuable benefits of therapy, such as morphine therapy. ${ }^{35}$ In order to perceive the clinically relevant improvement in more patients, the prompt assessment and the management of the side effects, which may outweigh the net benefits, are extremely important. ${ }^{3,35}$ Addressing breathlessness through a proactive palliative method had a positive impact not only on alleviating symptoms but also a potential benefit on the survival of COPD patients. ${ }^{36}$ In the case that self-reported breathlessness cannot be assessed, proxy measurements from caregivers specialized in palliative care can be used for symptom management. ${ }^{37}$

Palliative care is a holistic care focused on the whole person. ${ }^{3}$ Alongside the distressing symptom burden, the multidimensional authentic needs of patients living with $\mathrm{COPD}^{4}$ are other important barriers in offering PC integrated into the management of the disease, as early as the guidelines ideally advise. ${ }^{38}$ Early recognition of the timing for PC is necessary, but not sufficient, for effective care. ${ }^{33}$ The relationship between these unsatisfied needs, and prevention and management of the disease has been broadly addressed in the literature. ${ }^{39,40}$ Knowing the real dimensions in which COPD patients express their unsatisfied needs could lead to a better approach and care, even in individuals who overlook their needs. ${ }^{4}$ Simple open questions addressed by primary care clinicians or specialists are aimed not only to improve communication barriers, but also to explore these issues with their patients. ${ }^{15}$

On the other hand, the "surprise-question" (SQ) that physicians may ask themselves is: "Would I be surprised if my patients were to die in the next 12 months?" If the answer is "no," this may be seen as an additional screening tool for the identification of the patients who would benefit from early proactive palliative care. ${ }^{15,16,33}$ It is incorporated into clinical guidelines such as the National Institute for Health and Care Excellence (NICE) for End of Life Care $^{41}$ and included as part of the Gold Standard Framework (GSF) proactive identification guidance tool in the UK. ${ }^{42}$ Though the predictive power of the SQ varies among clinicians, settings, and seniority, ${ }^{43}$ its application in COPD was shown to be highly unreliable. ${ }^{17}$ Moreover, recent data outlined important limitations in the use of the surprise question and clinician-related approaches. ${ }^{44}$ Therefore, the role of the SQ should be carefully weighed by each physician. They should never forget that palliative 
care is appropriate for COPD patients at any point in their disease trajectory. ${ }^{16}$

\section{Study Limitations}

Understandably the present review has some limitations. The use of a single selected database and the limitation on the language search could have led to the exclusion of studies on the topic of palliative care and prognosis in COPD patients. Another limitation of the review was the fact that all the results were from papers published in industrialized countries, meaning that different sociocultures and health-care systems from the developing world were not represented and therefore the results may not be generalized. There is a need for larger prognostic studies, from different settings and forms of COPD, in order to limit the bias of the present review.

\section{Conclusion}

The barriers in offering timely integrated PC services to COPD patients are complex. Despite the identification of validated prognostic variables and multidimensional indices, none of them are sufficiently reliable to predict survival and implicit the moment to start PC in COPD patients. The decision to initiate $\mathrm{PC}$ should be rather based on the presence of refractory chronic symptoms and patients' unmet needs and preferences. Despite the current advances, the ideal model to initiate longitudinal palliative care from the moment COPD is diagnosed, alongside the usual management of the disease and intensified care in the end-of-life stages, is a goal for clinicians trained in and capable of providing palliative care in any COPD patient.

\section{Disclosure}

The authors report no conflicts of interest in this work.

\section{References}

1. Chronic respiratory diseases [homepage on the Internet]. Geneva: World Health Organization; 2020. Available from: https://www.who. int/health-topics/chronic-respiratory-diseases\#tab=tab_1. Accessed March 15, 2020.

2. Chuchalin AG, Khaltaev N, Antonov NS, et al. Chronic respiratory diseases and risk factors in 12 regions of the Russian Federation. Int J Chron Obstruct Pulmon Dis. 2014;9:963. doi:10.2147/COPD. S67283

3. Maddocks M, Lovell N, Booth S, Man WD, Higginson IJ. Palliative care and management of troublesome symptoms for people with chronic obstructive pulmonary disease. Lancet. 2017;390 (10098):988-1002. doi:10.1016/S0140-6736(17)32127-X

4. Clari M, Ivziku D, Casciaro R, Matarese M. The unmet needs of people with chronic obstructive pulmonary disease: a systematic review of qualitative findings. COPD. 2018;15(1):79-88. doi:10.1080/15412555.2017.1417373
5. Smith LJ, Moore E, Ali I, Smeeth L, Stone P, Quint JK. Prognostic variables and scores identifying the end of life in COPD: a systematic review. Int $J$ Chron Obstruct Pulmon Dis. 2017;12:2239. doi:10.2147/COPD.S137868

6. Public Health England. Palliative and end of life care: hospital deaths 2006 to 2017 [homepage on the Internet]; 2019. Available from: https://www.gov.uk/government/publications/palliative-and-end-oflife-care-hospital-deaths-2006-to-2017/palliative-and-end-of-life-care -hospital-deaths-2006-to-2017. Accessed March 15, 2020.

7. World Health Organization. Action plan of the Global alliance against chronic respiratory diseases, 2008-2013; 2008. Available from: https:// apps.who.int/iris/bitstream/handle/10665/43984/9789241597203_eng. pdf? sequence=1\&isAllowed=y. Accessed March 15, 2020.

8. Halpin DMG. Palliative care for chronic obstructive pulmonary disease. Signs of progress, but still a long way to go. Am J Respir Crit Care Med. 2018;198(11):1356-1358. doi:10.1164/rccm.2018050955ED

9. World Health Organization. WHO definition of palliative care. Available from: http://www.who.int/cancer/palliative/definition/en/. Accessed May 19, 2020.

10. Al-Mahrezi A, Al-Mandhari Z. Palliative care: time for action. Oman Med J. 2016;31(3):161-163. doi:10.5001/omj.2016.32

11. McVeigh C, Reid J, Carvalho P. Healthcare professionals' views of palliative care for American war veterans with non-malignant respiratory disease living in a rural area: a qualitative study. BMC Palliat Care. 2019;18(1):22. doi:10.1186/s12904-019-0408-7

12. Lanken PN, Terry PB, DeLisser HM, et al. An official American Thoracic Society clinical policy statement: palliative care for patients with respiratory diseases and critical illnesses. Am J Respir Crit Care Med. 2008;177(8):912-927. doi:10.1164/rccm.200605-587ST

13. Connor SR, Bermedo MC, editors. Global Atlas of Palliative Care at the End of Life. Worldwide Palliative Care Alliance; 2014.

14. Shamseer L, Moher D, Clarke M, et al. Preferred reporting items for systematic review and meta-analysis protocols (PRISMA-P) 2015: elaboration and explanation. BMJ. 2015;349:g7647. doi:10.1136/bmj.g7647

15. Vermylen JH, Szmuilowicz E, Kalhan R. Palliative care in COPD: an unmet area for quality improvement. Int J Chron Obstruct Pulmon Dis. 2015;10:1543. doi:10.2147/COPD.S74641

16. Harrington SE, Rogers E, Davis M. Palliative care and chronic obstructive pulmonary disease: where the lines meet. Curr Opin Pulm Med. 2017;23(2):154-160. doi:10.1097/MCP.0000000000000358

17. Almagro P, Yun S, Sangil A, et al. Palliative care and prognosis in COPD: a systematic review with a validation cohort. Int J Chron Obstruct Pulmon Dis. 2017;12:1721. doi:10.2147/COPD.S135657

18. Siouta N, van Beek K, Preston N, et al. Towards integration of palliative care in patients with chronic heart failure and chronic obstructive pulmonary disease: a systematic literature review of European guidelines and pathways. BMC Palliat Care. 2016;15 (1):18. doi:10.1186/s12904-016-0089-4

19. Parry A, Higginson R, Gleeson A. End-of-life prognostic indicators in patients with COPD: part 2. Int J Palliat Nurs. 2016;22 (11):560-567. doi:10.12968/ijpn.2016.22.11.560

20. Curtis JR. Palliative and end-of-life care for patients with severe COPD. Eur Respir J. 2008;32(3):796-803. doi:10.1183/ 09031936.00126107

21. Boeck L, Soriano JB, Brusse-Keizer M, et al. Prognostic assessment in COPD without lung function: the B-AE-D indices. Eur Respir $J$. 2016;47(6):1635-1644. doi:10.1183/13993003.01485-2015

22. Bloom CI, Ricciardi F, Smeeth L, Stone P, Quint JK. Predicting COPD 1-year mortality using prognostic predictors routinely measured in primary care. BMC Med. 2019;17(1):73. doi:10.1186/ s12916-019-1310-0

23. Ekström M, Currow DC, Johnson MJ. Outcome measurement of refractory breathlessness: endpoints and important differences. Curr Opin Support Palliat Care. 2015;9(3):238-243. doi:10.1097/ SPC.0000000000000147 
24. Leidy NK, Sexton CC, Jones PW, et al. Measuring respiratory symptoms in clinical trials of COPD: reliability and validity of a daily diary. Thorax. 2014;69(5):443-449. doi:10.1136/thoraxjnl-2013204428

25. Wilcox TK, Chen WH, Howard KA, et al. Item selection, reliability and validity of the Shortness of Breath with Daily Activities (SOBDA) questionnaire: a new outcome measure for evaluating dyspnea in chronic obstructive pulmonary disease. Health Qual Life Outcomes. 2013;11(1):196. doi:10.1186/1477-7525-11-196

26. Yorke J, Lloyd-Williams M, Smith J, et al. Management of the respiratory distress symptom cluster in lung cancer: a randomised controlled feasibility trial. Support Care Cancer. 2015;23 (11):3373-3384. doi:10.1007/s00520-015-2810-x

27. Banzett RB, O'Donnell CR, Guilfoyle TE, et al. Multidimensional Dyspnea profile: an instrument for clinical and laboratory research. Eur Respir J. 2015;45(6):1681-1691. doi:10.1183/ 09031936.00038914

28. Clark D. From margins to centre: a review of the history of palliative care in cancer. Lancet Oncol. 2007;8(5):430-438. doi:10.1016/S14702045(07)70138-9

29. Lilly EJ, Senderovich H. Palliative care in chronic obstructive pulmonary disease. J Crit Care. 2016;35:150-154. doi:10.1016/j. jcrc.2016.05.019

30. Bellou V, Belbasis L, Konstantinidis AK, Tzoulaki I, Evangelou E. Prognostic models for outcome prediction in patients with chronic obstructive pulmonary disease: systematic review and critical appraisal. BMJ. 2019;367:15358. doi:10.1136/bmj.15358

31. Puhan MA, Hansel NN, Sobradillo P, et al. Large-scale international validation of the ADO index in subjects with COPD: an individual subject data analysis of 10 cohorts. BMJ Open. 2012;2(6):e002152. doi:10.1136/bmjopen-2012-002152

32. Bertens LC, Reitsma JB, Moons KG, et al. Development and validation of a model to predict the risk of exacerbations in chronic obstructive pulmonary disease. Int J Chron Obstruct Pulmon Dis. 2013;8:493-499.

33. Murray SA, Boyd K, Sheikh A. Palliative care in chronic illness. BMJ. 2005;330(7492):611-612.

34. Yorke J, Swigris J, Russell AM, et al. Dyspnea-12 is a valid and reliable measure of bre Ferreira DH, Silva JP, Quinn S, et al. Blinded patient preference for morphine compared to placebo in the setting of chronic refractory breathlessness-an exploratory study. J Pain Symptom Manage. 2016;51(2):247-254.
35. Ferreira DH, Silva JP, Quinn S, et al. Blinded patient preference for morphine compared to placebo in the setting of chronic refractory breathlessness-an exploratory study. J Pain Symptom Manage. 2016;51(2):247-254. doi:10.1016/j.jpainsymman.2015.10.005

36. Higginson IJ, Bausewein C, Reilly CC, et al. An integrated palliative and respiratory care service for patients with advanced disease and refractory breathlessness: a randomised controlled trial. Lancet Respir Med. 2014;2(12):979-987. doi:10.1016/S2213-2600(14) 70226-7

37. Quinn C, Dunbar SB, Higgins M. Heart failure symptom assessment and management: can caregivers serve as proxy? J Cardiovasc Nurs. 2010;25(2):142. doi:10.1097/JCN.0b013e3181bf93a0

38. Nici L, Donner C, Wouters E, et al. American thoracic society/ European respiratory society statement on pulmonary rehabilitation. Am J Respir Crit Care Med. 2006;173(12):1390-1413. doi:10.1164/ rccm.200508-1211ST

39. Gruffydd-Jones K, Langley-Johnson C, Dyer C, Badlan K, Ward S. What are the needs of patients following discharge from hospital after an acute exacerbation of chronic obstructive pulmonary disease (COPD)? Prim Care Respir J. 2007;16(6):363-368. doi:10.3132/ pcrj.2007.00075

40. Wong SS, Abdullah N, Abdullah A, et al. Unmet needs of patients with chronic obstructive pulmonary disease (COPD): a qualitative study on patients and doctors. BMC Fam Pract. 2014;15(1):6. doi:10.1186/1471-2296-15-67

41. National Institute for Health and Care Excellence. End of life care for adults; 2011. Available from: https://www.nice.org.uk/guidance/qs13. Accessed May 20, 2020.

42. Thomas K, Armstrong Wilson J; GSF Team. Proactive identification guidance (PIG) national gold standards framework centre in end of life care; 2016. Available from: https:/www.nice.org.uk/guidance/ qs13. Accessed May 19, 2020.

43. Da Silva Gane M, Braun A, Stott D, Wellsted D, Farrington K. How robust is the 'surprise question' in predicting short-term mortality risk in haemodialysis patients? Nephron Clin Pract. 2013;123(3-4):185-193. doi:10.1159/000353735

44. Downar J, Wegier P, Tanuseputro P. Early identification of people who would benefit from a palliative approach-moving from surprise to routine. JAMA Netw Open. 2019;2(9):e1911146. doi:10.1001/ jamanetworkopen.2019.11146

\section{Publish your work in this journal}

The International Journal of COPD is an international, peer-reviewed journal of therapeutics and pharmacology focusing on concise rapid reporting of clinical studies and reviews in COPD. Special focus is given to the pathophysiological processes underlying the disease, intervention programs, patient focused education, and self management protocols. This journal is indexed on PubMed Central, MedLine and CAS. The manuscript management system is completely online and includes a very quick and fair peer-review system, which is all easy to use. Visit http://www.dovepress.com/testimonials.php to read real quotes from published authors. 